\title{
Análisis de la mortalidad por leucemia aguda pediátrica en el Instituto Nacional de Cancerología
}

\author{
Anyul Milena Vera ${ }^{1}$, Constanza Pardo¹, María Cristina Duarte², Amaranto Suárez ${ }^{3}$ \\ 1 Grupo de Vigilancia Epidemiológica del Cáncer, Instituto Nacional de Cancerología, E.S.E., Bogotá, D.C., \\ Colombia \\ 2 E.S.E. Hospital San Cristóbal, Bogotá, D.C., Colombia \\ ${ }^{3}$ Clínica de Oncología Pediátrica, Instituto Nacional de Cancerología, E.S.E., Bogotá, D.C., Colombia
}

Introducción. La mortalidad por leucemia aguda pediátrica es un indicador trazador del acceso y efectividad de la atención en salud; su análisis permite identificar los factores involucrados en el proceso de la enfermedad que pueden influir en la condición final de los niños.

Objetivo. Describir la experiencia operativa y los resultados obtenidos en los comités de análisis institucional de la mortalidad por leucemia aguda pediátrica.

Materiales y métodos. Se hizo un análisis descriptivo de las muertes por leucemia linfoide o mieloide aguda ocurridas en niños menores de 15 años en el Instituto Nacional de Cancerología, 2008-2010. Se llevó a cabo el análisis de "evitabilidad" (sic.) con el modelo de las tres demoras propuesto por Thaddeus y Maine.

Resultados. Se analizaron 24 defunciones. El 87,5 \% fueron a causa de leucemia linfoide aguda. La mediana de edad fue de 10,5 años. Se encontró que la demora 3 (obtener el tratamiento adecuado y oportuno) ocurrió en el $83 \%$ de los casos analizados y que los traslados durante el tratamiento fue la limitación más común $(66,7 \%$ ). El $83,3 \%$ de las muertes se consideraron no evitables dadas las condiciones clínicas de la enfermedad.

Conclusiones. Es la primera experiencia en el análisis de mortalidad por un evento crónico en la población pediátrica del Instituto Nacional de Cancerología y en el país. Aunque las demoras identificadas no condicionaron de forma directa la mortalidad, sí constituyen la base para establecer acciones de mejoría que influyan en la calidad de la atención de los niños con cáncer.

Palabras clave: leucemia/mortalidad, mortalidad infantil, salud pública, vigilancia sanitaria, Colombia. doi: http://dx.doi.org/10.7705/biomedica.v32i3.691

\section{Analysis of mortality in pediatric acute leukemia at the National Cancer Institute}

Introduction. Mortality rate for childhood acute leukemia is an indicator of access to and efficacy of health services. Analysis of this indicator allows the identification of factors contributing to the development of the illness and the final condition of affected children.

Objective. The operative experience results were described from data obtained by committee of analysis of institutional mortality by childhood acute leukaemia.

Materials and methods. Formal reports of deaths in children under 15 due to acute lymphoblastic or myeloid leukemia were provided to the National Cancer Institute of Colombia. A descriptive analysis of these reports from the period 2008-2010 was undertaken by the National Cancer Institute. Avoidability analysis was performed using the 1994 "three delays" model of Thaddeus and Maine.

Results. Of 24 deaths analyzed, 21 were caused by acute lymphoblastic leukemia. The median age was 10.5 years. The delay 3 (get adequate and timely treatment) was identified in $83 \%$ of the cases and transfers during treatment were the most common limitation (66.7\%). $83.3 \%$ of deaths were deemed unavoidable given the clinical conditions of the patients.

Conclusions. Even though the delays identified did not affect mortality in a direct way, they did nonetheless constitute the basis for improvements to the quality of attention to children with cancer.

\footnotetext{
Contribución de los autores:

Anyul Milena Vera: concepción y diseño del estudio, revisión de literatura, análisis e interpretación de datos, redacción del manuscrito y aprobación de la versión final.

Constanza Pardo: concepción y diseño del estudio, interpretación de datos, contribuyó a la redacción y revisión del manuscrito y aprobación de la versión final.

María Cristina Duarte: revisión de la metodología, contribuyó en la redacción de la discusión del manuscrito y aprobación de la versión final.

Amaranto Suárez: contribución médico-científico en la redacción de la discusión del manuscrito y aprobación de versión final.
} 
Key words: leukaemia/mortality, infant mortality, public health, health surveillance, Colombia.

doi: http://dx.doi.org/10.7705/biomedica.v32i3.691

El cáncer en los niños es una enfermedad rara que sólo representa entre 0,5 y $3 \%$ de todas las neoplasias malignas en el mundo; sin embargo, constituye un importante problema de salud pública, por la alta probabilidad de muerte a edades tempranas y por el impacto social en los niños, sus padres y sus familias. En general, las leucemias son la principal causa de cáncer pediátrico, seguidas de los tumores malignos del sistema nervioso central y los linfomas $(1,2)$, y corresponden al $30 \%$ de las neoplasias malignas que se presentan en menores de 15 años; de estas, aproximadamente, el $75 \%$ son leucemia linfoide aguda y del 15 al $20 \%$ son leucemia mieloide aguda (3). En Colombia, se estima que en este grupo de edad ocurren 497 casos nuevos de leucemia cada año (2), lo que representa el $42,5 \%$ de todos los tipos de cáncer en la niñez, con una distribución mayor en niños que en niñas.

En los países desarrollados, la mortalidad por leucemia infantil ha disminuido de manera importante en los últimos 30 años, mientras que la incidencia ha permanecido sin cambios $(4,5)$. En los países en desarrollo, la incidencia se mantiene, pero las tasas de mortalidad por leucemia pediátrica continúan siendo altas (6). En Colombia, para el 2008, el $48,2 \%$ de las defunciones por cáncer en menores de 15 años correspondió a leucemias de cualquier tipo (7).

La supervivencia a cinco años de los niños con leucemia linfoide aguda es del $85 \%$, en la mayoría de países europeos y en los Estados Unidos (8). En Cali (Colombia), la supervivencia observada a cinco años fue de $41 \%$ (1).

Algunos factores determinantes en el pronóstico de los niños con leucemias agudas, están relacionados con la biología, los subtipos de leucemia y los factores de índole social $(9,10)$. Estos últimos han marcado diferencias en los países en desarrollo, donde existen dificultades para el diagnóstico e inicio del tratamiento oportuno con el consecuente fracaso del tratamiento. Las principales causas de falla del tratamiento incluyen: la recaída, la muerte por toxicidad y el abandono o interrupción del tratamiento $(11,12)$.

\section{Correspondencia:}

Anyul Milena Vera, Grupo de Vigilancia Epidemiológica del Cáncer, Instituto Nacional de Cancerología, E.S.E., Calle $1 \mathrm{~N}^{\circ}$ 9-85, Bogotá, D.C., Colombia

Teléfono: (571) 334 1111, extensión 4108

amvera@cancer.gov.co

Recibido: 20/06/11; aceptado:22/03/12
La letalidad es un indicador de las diferencias en el acceso, el tipo de tratamiento y las condiciones socioeconómicas de los niños con leucemia aguda. La razón incidencia/mortalidad estimada para Colombia en 2002 fue de 1,3, mientras que para Estados Unidos fue de 5,1 (11), lo que indica una alta letalidad en nuestro medio.

Dado el comportamiento de las leucemias agudas pediátricas en el país y con base en la ley de infancia y adolescencia (13), el Ministerio de la Protección Social decidió abordar el problema de forma prioritaria. A partir de 2008 se implementó un sistema de vigilancia centinela de las leucemias agudas pediátricas, para identificar los casos en menores de 15 años y garantizar la oportunidad en el diagnóstico y en el inicio del tratamiento (14), en el marco del Sistema de Vigilancia en Salud Pública (Sivigila) (15).

Para las muertes por leucemias agudas pediátricas se definió por protocolo, el estudio y el análisis de la posibilidad de evitar ("evitabilidad") (sic.) de cada muerte ocurrida (16). Esta estrategia permite identificar los factores involucrados en el proceso de atención de la enfermedad y en la condición final de los niños. Históricamente, los análisis de mortalidad en niños se han orientado a las enfermedades infectocontagiosas, inmunoprevenibles y perinatales $(17,18)$. Esta es la primera experiencia para un evento crónico en este grupo de edad.

Este artículo presenta los resultados del análisis de la mortalidad por leucemias agudas pediátricas en menores de 15 años, ocurridas en el Instituto Nacional de Cancerología, 2008 a 2010, y la divulgación de la experiencia con la metodología implementada, como ejemplo para otras instituciones de salud del país que tienen como responsabilidad el desarrollo de estos comités de análisis.

\section{Materiales y métodos}

Se hizo un estudio descriptivo de corte transversal en el que se analizaron todas las defunciones por leucemia linfoide o mieloide aguda, en niños menores de 15 años, ocurridas en el Instituto Nacional de Cancerología y certificadas por médicos de la entidad durante el periodo 2008-2010.

\section{Fuentes de información}

Se utilizaron los certificados de defunción y las fichas epidemiológicas para la notificación del 
evento al Sivigila (19) generados por el Instituto Nacional de Cancerología y las actas emitidas por los comités de análisis de la mortalidad por leucemias agudas pediátricas.

\section{Actividades previas al comité de análisis de la mortalidad por leucemias agudas pediátricas}

Se hizo una búsqueda activa de las muertes en las bases de datos de mortalidad institucional para el periodo definido. Para cada muerte se diligenció la ficha epidemiológica para leucemia linfoide o mieloide aguda pediátrica, establecida por el Sivigila (19).

Una vez identificado el caso, se enviaron la ficha epidemiológica, el resumen de historia clínica y la copia del certificado de defunción a la unidad notificadora local E.S.E. Hospital San Cristóbal.

La unidad notificadora remitió la información de la muerte por leucemia aguda pediátrica a la
Dirección Territorial de Salud correspondiente y convocó a cada una de las instituciones involucradas en el proceso de atención del niño. Se invitó a los representantes de las Instituciones Prestadoras de Servicios de Salud, las Empresas Administradoras de Planes de Beneficios, al Grupo de Enfermedades Crónicas de la Secretaría Distrital de Salud de Bogotá y al Grupo de Epidemiología del Hospital de Primer Nivel del lugar de residencia del fallecido. El Grupo de Enfermedades Crónicas del Instituto Nacional de Salud fue convocado para los comités de análisis de muertes por leucemias agudas pediátricas de pacientes con lugar de residencia distinto a Bogotá (cuadro 1).

\section{Comité de análisis de la mortalidad por leucemias agudas pediátricas}

Para la divulgación del caso, se hicieron lecturas cronológicas de los procesos de atención ofrecidos por las instituciones intervinientes, desde la

Cuadro 1. Actividades desarrolladas por las instituciones participantes de los comités de análisis de las muertes por leucemias agudas pediátricas, Instituto Nacional de Cancerología

\begin{tabular}{|c|c|}
\hline Institución & Actividades \\
\hline \multirow[t]{4}{*}{ Instituto Nacional de Cancerología } & Identifica la mortalidad por leucemias agudas pediátricas en el certificado de defunción. \\
\hline & Diligencia la ficha epidemiológica y se hace la notificación en archivo plano al Sivigila. \\
\hline & $\begin{array}{l}\text { Remite la ficha epidemiológica, el resumen de historia clínica y el certificado de defunciór } \\
\text { a la Unidad Notificadora- Hospital San Cristóbal. }\end{array}$ \\
\hline & $\begin{array}{l}\text { Asiste al comité de análisis de mortalidad como institución involucrada en el proceso de } \\
\text { atención. }\end{array}$ \\
\hline \multirow{4}{*}{$\begin{array}{l}\text { Empresa Social del Estado Hospital } \\
\text { San Cristóbal }\end{array}$} & Reporta el caso a la Dirección Territorial de Salud (Bogotá). \\
\hline & $\begin{array}{l}\text { Reporta el caso a la Dirección Territorial de Salud del departamento de residencia del } \\
\text { fallecido. }\end{array}$ \\
\hline & $\begin{array}{l}\text { Convoca a las instituciones involucradas en el proceso de atención (otras instituciones } \\
\text { prestadoras de servicios de salud y empresas administradoras de planes de beneficios) } \\
\text { al comité de análisis de mortalidad por leucemias agudas pediátricas. }\end{array}$ \\
\hline & Realiza el comité de análisis de mortalidad \\
\hline $\begin{array}{l}\text { Hospital público de primer nivel } \\
\text { en lugar de residencia del fallecido }\end{array}$ & $\begin{array}{l}\text { Realiza la investigación epidemiológica de campo. } \\
\text { Asiste al comité de análisis de mortalidad como institución involucrada en el proceso de } \\
\text { atención. }\end{array}$ \\
\hline $\begin{array}{l}\text { Empresas administradoras de planes } \\
\text { de beneficios }\end{array}$ & $\begin{array}{l}\text { Generan el resumen de atenciones al paciente. } \\
\text { Asisten al comité de análisis de mortalidad como institución involucrada en el proceso } \\
\text { de atención. }\end{array}$ \\
\hline $\begin{array}{l}\text { Otras instituciones prestadoras de } \\
\text { servicios de salud }\end{array}$ & $\begin{array}{l}\text { Generan el resumen de atenciones al paciente. } \\
\text { Asisten al comité de análisis de mortalidad como institución involucrada en el proceso } \\
\text { de atención. }\end{array}$ \\
\hline $\begin{array}{l}\text { Grupo de Enfermedades Crónicas de } \\
\text { la Secretaría Distrital de Salud de } \\
\text { Bogotá }\end{array}$ & $\begin{array}{l}\text { Participa en los comités de análisis de muertes por leucemias agudas pediátricas en } \\
\text { niños residentes en Bogotá, D.C. }\end{array}$ \\
\hline $\begin{array}{l}\text { Grupo de Enfermedades Crónicas del } \\
\text { Instituto Nacional de Salud }\end{array}$ & $\begin{array}{l}\text { Participa en los comités de análisis de muertes por leucemias agudas pediátricas en } \\
\text { niños residentes en municipios diferentes a Bogotá, D.C. }\end{array}$ \\
\hline
\end{tabular}


definición de caso probable hasta la presentación de la muerte. En algunos casos hubo disponibilidad del informe de la investigación epidemiológica de campo (20), construido a partir de una entrevista semiestructurada a la familia del menor fallecido; por lo anterior, los elementos obtenidos fueron limitados y no se incluyeron en el consolidado general de los resultados de tipo cuantitativo, evitando sesgos de información.

Finalmente, se revisó la cadena causal del certificado de defunción y se generó un espacio de discusión para emitir conclusiones y compromisos consignados en actas independientes para cada caso, con la identificación de aspectos por intervenir, y la generación de planes de mejora y recomendaciones para las instituciones en las que se identificaron falencias.

\section{Análisis de datos}

El análisis de las muertes se apoyó en el modelo de Thaddeus y Maine (21), validado en el análisis de la mortalidad materna (22) pero aplicado, en la actualidad, para el estudio de la mortalidad evitable en otros eventos de interés en salud pública (23). El modelo permitió identificar los factores corresponsables de la muerte, en función de las demoras ocurridas durante el proceso de atención de la enfermedad y responder a la pregunta ¿por qué murió el individuo?, obteniendo elementos para el análisis crítico de cada situación enmarcado en el contexto del sistema de salud actual.

Las demoras se describieron en tres grupos no excluyentes entre sí, permitiendo que un niño tuviera simultáneamente más de una demora:

1) Demora en decidir buscar atención, la cual analiza la capacidad de la madre, el padre y los miembros del entorno para reconocer signos y síntomas que amenazan la vida y la disponibilidad de información para decidir a qué sitio acudir en busca de ayuda; además, analiza el cumplimiento del tratamiento y la demora para consultar con urgencia durante el tratamiento.

2) Demora en identificar $y$ acceder a un servicio de salud, mediante la cual se analizan las dificultades para llegar a los servicios de salud, $\mathrm{y}$

3) Demora en obtener el tratamiento adecuado y oportuno, en la cual se analiza la disponibilidad de personal entrenado con el que cuentan las instituciones desalud, asícomolainfraestructura, disponibilidad de equipos y medicamentos, incluyendo los tiempos para la confirmación diagnóstica y el inicio del tratamiento (21).

Además, durante los análisis se incluyó un componente con preguntas que buscaban identificar las causas biológicas y clínicas de la muerte. A partir de los análisis de las demoras y del componente biológico-clínico, se emitió una conclusión sobre la posibilidad de haber evitado ("evitabilidad") (sic.) de la muerte.

Se generó una matriz de variables en la que se unificaron los criterios evaluados y extraidos de cada uno de los casos revisados en los comités de análisis. Se calculó la variable "tiempo trascurrido entre el reconocimiento de signos de alarma y la consulta a una institución de salud" a partir de la fecha del inicio de síntomas y la fecha de la consulta. El tiempo transcurrido entre el diagnóstico probable y el diagnóstico confirmado, y el tiempo entre la confirmación y el inicio del tratamiento, se obtuvieron de las fichas epidemiológicas.

Para el análisis de los datos se utilizó el paquete estadístico SPSS ${ }^{\text {TM }}$, versión 18. Para el análisis descriptivo se usaron medidas de tendencia central y de dispersión para las variables cuantitativas, y proporciones para las variables cualitativas.

\section{Resultados}

De las 24 defunciones analizadas para el periodo 2008-2010, el 87,5 \% tuvo como causa básica la leucemia linfoide aguda y la mitad ocurrieron en el 2010. Se observó que las muertes fueron más frecuentes en el sexo masculino y en menores procedentes de la cabecera municipal. El $58 \%$ de los niños residía en Bogotá y estaban afiliados al régimen subsidiado (cuadro 2). La mediana de edad para ambos sexos fue de 10,5 años. Al $33,3 \%$ de las muertes se les realizó la investigación epidemiológica de campo.

En el análisis por demoras se identificó que la demora 3 (obtener el tratamiento adecuado y oportuno) fue la más común entre las 24 muertes analizadas (figura 1).

Al analizar los elementos incluidos en la demora 1 (decidir buscar atención), se encontró que la mediana de tiempo transcurrido entre el reconocimiento de los signos de alarma por los padres y la consulta a una institución de salud, fue de 8 días; sólo el $29 \%$ de los casos consultó luego de 15 días de la aparición de los síntomas (cuadro 3). En el cumplimiento, se identificó que seis de los casos abandonaron en algún momento el tratamiento y la 
Cuadro 2. Características socio-demográficas de los menores de 15 años con leucemias agudas pediátricas, Instituto Nacional de Cancerología, 2008-2010

\begin{tabular}{|c|c|c|c|}
\hline Variable & Niveles de la variable & $\mathbf{n}$ & $\%$ \\
\hline \multirow[t]{2}{*}{ Diagnóstico } & Leucemia linfoide aguda & 21 & 87,5 \\
\hline & Leucemia mieloide aguda & 3 & 12,5 \\
\hline \multirow{3}{*}{ Año de defunción } & 2008 & 4 & 16,7 \\
\hline & 2009 & 8 & 33,3 \\
\hline & 2010 & 12 & 50 \\
\hline \multirow{3}{*}{ Edad (años) } & $<5$ & 3 & 12,5 \\
\hline & 5 a 10 & 9 & 37,5 \\
\hline & Hasta 15 & 12 & 50 \\
\hline \multirow[t]{2}{*}{ Sexo } & Femenino & 9 & 37,5 \\
\hline & Masculino & 15 & 62,5 \\
\hline \multirow{3}{*}{ Área de residencia } & Cabecera municipal & 19 & 79,2 \\
\hline & Centro poblado & 1 & 4,2 \\
\hline & Rural disperso & 4 & 16,7 \\
\hline \multirow{5}{*}{ Lugar de residencia } & Bogotá, D.C. & 14 & 58,3 \\
\hline & Boyacá & 4 & 16,7 \\
\hline & Cundinamarca & 4 & 16,7 \\
\hline & Huila & 1 & 4,2 \\
\hline & Tolima & 1 & 4,2 \\
\hline \multirow{3}{*}{ Régimen de afiliación en salud } & Contributivo & 3 & 12,5 \\
\hline & Subsidiado & 12 & 50 \\
\hline & No asegurado & 9 & 37,5 \\
\hline
\end{tabular}

principal razón de abandono fue la percepción de curación por parte de la familia y, en consecuencia, la inasistencia a las citas programadas. Para la consulta a urgencias, sólo se encontró un caso que no acudió de manera oportuna tras un pico febril posterior a la quimioterapia, hecho que condicionó el desenlace fatal (cuadro 3).

En la demora 2 (identificar y acceder a un servicio de salud), se identificaron 12 niños que se desplazaron a otras ciudades para recibir diagnóstico y tratamiento, situación debida a la carencia de infraestructura hospitalaria y la baja complejidad de los centros asistenciales en sus lugares de residencia (cuadro 3).

Para la demora 3 (obtener el tratamiento adecuado y oportuno), se dedujo que el $83 \%$ de los niños tuvo alguna limitación de este tipo (figura 1). Además, se encontró que el tiempo promedio transcurrido entre el diagnóstico probable y el diagnóstico confirmado fue de cinco días; entre tanto, el tiempo promedio entre la confirmación y el inicio del tratamiento fue de tres días (cuadro 3). La principal limitación durante el tratamiento fue el traslado de los niños entre Instituciones Prestadoras de Servicios de Salud; en los comités de análisis se encontró como posibles causas la inestabilidad contractual de las Empresas Administradoras de Planes de Beneficios con la red prestadora de servicios y la deficiencia

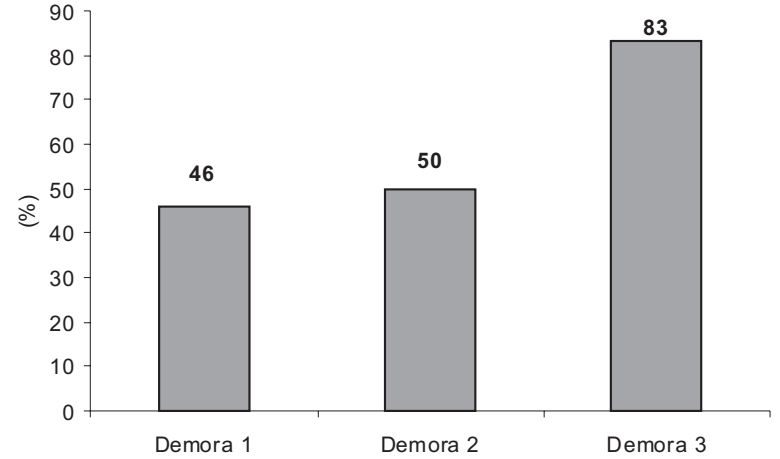

Figura 1. Distribución de las demoras que pudieron influir en la muerte por leucemia aguda pediátrica, Instituto Nacional de Cancerología, 2008-2010

en la infraestructura hospitalaria para la atención a pacientes oncológicos. Se identificaron otros problemas relacionados con el aseguramiento (58 $\%)$, como inconsistencias en las bases de datos de afiliados, deficiencias en el proceso de referencia y contrarreferencia, y poca disponibilidad de puntos de atención para solicitud de autorizaciones, lo que implicó largos desplazamientos (cuadro 3).

El análisis de posibilidades de evitar la muerte ("evitabilidad") (sic.) en función de la condición clínica de los niños, mostró que $83,3 \%$ de las defunciones ocurridas eran muertes inevitables y se destacó la recaída de la enfermedad como la 
principal causa relacionada con el desenlace. Para las muertes evitables, se encontró que los casos correspondían a menores con buena respuesta al tratamiento o en fase de remisión, pero que habían presentado alguna situación clínica (cuadro 4) o administrativa que condicionó la muerte. Finalmente, se encontró que el tiempo promedio de seguimiento en cada niño desde el diagnóstico hasta la muerte fue de 29 meses.

Fortalezas y limitaciones en el desarrollo de los comités de análisis de la mortalidad por leucemias agudas pediátricas

Como primera experiencia en el Instituto Nacional de Cancerología y en el país, se encontraron fortalezas, como la conformación de un equipo multidisciplinario, la respuesta aceptable a la convocatoria de los entes involucrados en la atención de los niños, el conocimiento y la apropiación del protocolo de vigilancia de las leucemias agudas pediátricas, y la mayor concientización de las implicaciones del manejo clínico y administrativo de un paciente pediátrico con cáncer. Las limitaciones en el desarrollo de los comités de análisis fueron punto de partida para la generación e implementación progresiva de acciones de mejora en el Instituto Nacional de Cancerología y en las entidades que participaron en los comités (cuadro 5).

\section{Discusión}

Este es un primer acercamiento al desarrollo de los comités de análisis de la mortalidad institucional por un evento crónico y catastrófico en la población infantil, como son las leucemias agudas pediátricas. La adaptación y aplicación de la metodología reconocida para la vigilancia de la mortalidad materna y otros eventos agudos $(18,21)$, permitieron identificar condiciones clínicas, sociales y administrativas que a lo largo del proceso de enfermedad pudieron haber afectado la oportunidad en la atención y condicionar la muerte por leucemia aguda en menores de 15 años.

Los resultados sociodemográficos de este estudio son concordantes con la situación observada en América Latina y en Colombia, con las leucemias como primera causa de muerte en menores de 15 años y predominio de la variante linfoide en la población masculina $(1,24)$.

La herramienta de investigación epidemiológica de campo se consideró como la "voz de la familia" en

Cuadro 3. Descripción de las demoras encontradas en las muertes por leucemias agudas pediátricas, Instituto Nacional de Cancerología, 2008-2010

\begin{tabular}{|c|c|c|c|c|}
\hline Demora & Variable & Niveles de la variable & $\mathbf{n}$ & $\%$ \\
\hline \multirow[t]{9}{*}{ Demora 1} & \multirow{5}{*}{$\begin{array}{l}\text { Tiempo trascurrido (días) entre el } \\
\text { reconocimiento de signos de alarma } \\
\text { y la consulta a una institución de salud } \\
\text { Problemas de cumplimiento del tratamiento }\end{array}$} & Entre 1 y 7 & 11 & 46 \\
\hline & & Entre 8 y 14 & 6 & 25 \\
\hline & & Mayor de 15 & 7 & 29 \\
\hline & & Sí & 6 & 25 \\
\hline & & No & 18 & 75 \\
\hline & \multirow{4}{*}{$\begin{array}{l}\text { Causas de incumplimiento del } \\
\text { tratamiento }\end{array}$} & Percepción de curación & 3 & 50 \\
\hline & & Elección de tratamiento alternativo & 1 & 17 \\
\hline & & Escasez de recursos & 1 & 17 \\
\hline & & No ingestión de los medicamentos en casa & 1 & 17 \\
\hline \multirow[t]{4}{*}{ Demora 2} & \multirow[t]{2}{*}{ Problemas de accesibilidad geográfica } & Sí & 12 & 50 \\
\hline & & No & 12 & 50 \\
\hline & \multirow[t]{2}{*}{ Falta de infraestructura } & Sí & 11 & 45,8 \\
\hline & & No & 13 & 54,2 \\
\hline \multirow[t]{12}{*}{ Demora 3} & \multirow[t]{2}{*}{ Demora en la generación de autorizaciones } & Sí & 6 & 25,0 \\
\hline & & No & 18 & 75,0 \\
\hline & \multirow[t]{2}{*}{ Traslado entre IPS durante el tratamiento } & Sí & 16 & 66,7 \\
\hline & & No & 8 & 33,3 \\
\hline & \multirow[t]{2}{*}{ Interposición de tutela para obtener atención } & Sí & 3 & 12,5 \\
\hline & & No & 21 & 87,5 \\
\hline & \multirow[t]{2}{*}{ Otros problemas relacionados con el aseguramiento } & Sí & 14 & 58,3 \\
\hline & & No & 10 & 41,7 \\
\hline & \multirow[t]{2}{*}{ Demora (días) en la confirmación diagnóstica } & Mayor de 8 & 5 & 20,8 \\
\hline & & Menor o igual a 8 & 19 & 79,2 \\
\hline & \multirow[t]{2}{*}{ Demora (días) para el inicio del tratamiento } & Mayor de 2 & 5 & 20,8 \\
\hline & & Menor o igual a 2 & 19 & 79,2 \\
\hline
\end{tabular}


Cuadro 4. Análisis de evitabilidad clínica de las muertes por leucemias agudas pediátricas, Instituto Nacional de Cancerología, 2008-2010

\begin{tabular}{lcclrr}
\hline Mortalidad evitable & $\mathbf{n}$ & $\%$ & \multicolumn{1}{c}{ Causas } & $\mathbf{n}$ & $\%$ \\
\hline Sí & \multirow{2}{*}{4} & \multirow{2}{*}{16,7} & Infección en el tercer día después de quimioterapia & 2 & 50 \\
& & & El pronóstico se enmarcó en la tercera demora & 1 & 25 \\
& & & Otra causa de muerte, diferente a la leucemias agudas pediátricas & 1 & 25 \\
\hline No & \multirow{2}{*}{20} & \multirow{2}{*}{83,3} & Enfermedad concomitante & 1 & 5 \\
& & & Nunca hizo la remisión & 2 & 10 \\
& & & Recaída & 16 & 80 \\
& & & Toxicidad por tratamiento & 1 & 5 \\
\hline
\end{tabular}

Cuadro 5. Limitaciones y propuestas de mejora para los comités de análisis de las muertes por leucemias agudas pediátricas, Instituto Nacional de Cancerología

Limitación
Propuesta de mejora

Aclaración de roles para cada institución:

Confusión en el rol de las instituciones frente a la metodología de los comités de análisis de muertes por leucemias agudas pediátricas
Instituto Nacional de Cancerología, análisis técnico científico del proceso de enfermedad y coordinación logística de la reunión. E.S.E. Hospital San Cristóbal, liderazgo metodológico que incluye citar la reunión, comunicación entre actores, presidir la reunión, generación de actas para revisión y recepción de planes de mejora. Secretaría Distrital de Salud, garante del cumplimiento de las acciones contempladas en la norma.

\begin{tabular}{|c|c|}
\hline $\begin{array}{l}\text { No participación de los representantes de las } \\
\text { Instituciones Prestadoras de Salud o Direcciones } \\
\text { Territoriales para menores no residentes o que } \\
\text { recibieron atención en instituciones de municipios } \\
\text { diferentes a Bogot,á D.C. }\end{array}$ & $\begin{array}{l}\text { Convocatoria de un representante del Instituto Nacional de Salud } \\
\text { Comunicación con otros municipios mediante teleconferencia } \\
\text { Intermediación con el Instituto Nacional de Salud para contactar } \\
\text { representantes de instituciones de municipios diferentes a Bogotá, D.C. }\end{array}$ \\
\hline Baja asistencia a las reuniones & Citación a la reunión desde la E.S.E. Hospital San Cristóbal \\
\hline $\begin{array}{l}\text { Desconocimiento del protocolo y la obligatoriedad } \\
\text { en la vigilancia del evento }\end{array}$ & $\begin{array}{l}\text { Difusión del protocolo en cada reunión. } \\
\text { Solicitud de difusión del protocolo por los entes territoriales. }\end{array}$ \\
\hline $\begin{array}{l}\text { No disponibilidad de investigación epidemiológica de } \\
\text { campo }\end{array}$ & $\begin{array}{l}\text { Divulgación de protocolo por el referente de la Secretaría Distrital de } \\
\text { Salud a los hospitales de la red pública de Bogotá, D.C. } \\
\text { Solicitud de la investigación epidemiológica de campo mediante oficio a } \\
\text { la institución responsable. }\end{array}$ \\
\hline $\begin{array}{l}\text { Escaso cumplimiento de las recomendaciones y } \\
\text { planes de mejora }\end{array}$ & $\begin{array}{l}\text { Remisión de copia de las actas que incluya los compromisos derivados } \\
\text { del comité de análisis a la Superintendencia Nacional de Salud. }\end{array}$ \\
\hline
\end{tabular}

el comité de análisis y, aunque tuvo disponibilidad limitada, fue relevante para reconocer aspectos sociales que influyeron en el curso de la enfermedad, así como la percepción de la familia. Aunque solo la tercera parte de los análisis tuvo investigación epidemiológica de campo, en estos se observaron aspectos que tuvieron impacto en la dinámica familiar por la enfermedad crónica del niño, tales como alteración en la interrelación de los miembros, la disolución del núcleo familiar dado por los traslados entre municipios para el tratamiento, renuncia o pérdida del empleo, aislamiento social y deserción escolar $(25,26)$.

En el análisis de la segunda y la tercera demora, aproximadamente en el $80 \%$ de los casos, los tiempos se ajustaron a los establecidos en el protocolo para la vigilancia centinela de las leucemias agudas pediátricas (19). Sin embargo, en los tiempos no ajustados algunos factores pudieron interferir en el proceso de atención de los niños, como el lugar de residencia, la infraestructura hospitalaria y la tramitología impuesta por las entidades dentro del Sistema General de Seguridad Social en Salud que rige al país.

Como ejemplo de lo anterior, la tercera parte de los niños se encontraban sin afiliación al Sistema General de Seguridad Social en Salud, lo que indirectamente pone de manifiesto las menores posibilidades para tomar la decisión de consultar, de acceder al sistema de salud -factor crítico en el comportamiento de la mortalidad- (24), y de recibir un diagnóstico y tratamiento oportunos para 
una enfermedad considerada de alta complejidad. Cabe resaltar que una vez el niño entró como vinculado al Sistema General de Seguridad Social en Salud pudo obtener una cobertura integral en la atención.

Un problema evidente fue el alto porcentaje de niños que recibieron traslados entre una y otra Institución Prestadora de Servicios de Salud a lo largo de su proceso de enfermedad, lo cual refleja el fraccionamiento de los servicios y pone en evidencia a las entidades que no facilitaron a sus pacientes la atención integral en la Institución Prestadora de Servicios de Salud donde se generó el diagnóstico definitivo y se inició el tratamiento.

Aunque se consideró que $83,3 \%$ de las muertes eran inevitables como consecuencia del curso clínico de la enfermedad, es relevante que la demora 3 está presente en más de $80 \%$ de los casos. Por lo anterior, la mortalidad por leucemia aguda pediátrica se debe reconocer como un problema social en la medida que está relacionada con el entorno político y económico, así como por factores sociales macroestructurales $(24,27)$, que trascienden los factores exclusivamente médicos y llevan a reflexionar sobre las repercusiones de la iniquidad en la atención de pacientes con cáncer (9).

El sistema de vigilancia centinela de las leucemias agudas pediátricas ha permitido un avance en el conocimiento de los casos nuevos y muertes por este evento en el país (16). No obstante, el objetivo fundamental del protocolo de vigilancia para leucemias agudas pediátricas, es evaluar la oportunidad en la atención y a partir de esta información diseñar estrategias de control y mejorar el funcionamiento de los actores del Sistema General de Seguridad Social en Salud frente a los problemas identificados.

La mortalidad por leucemias agudas pediátricas se considera como un indicador trazador del acceso y la efectividad de la atención médica especializada (28), que refleja las dificultades en el acceso a un diagnóstico y tratamiento oportunos, deficiencias en los cuidados de soporte y atención en centros hospitalarios que no cuentan con infraestructura para garantizar el manejo integral y protocolizado que requiere el niño con cáncer. Los estudios demuestran que el fortalecimiento de estos aspectos disminuye de forma importante la recaída, el abandono, la mortalidad asociada a toxicidad y, finalmente, aumentan la supervivencia; ejemplo de lo anterior es la experiencia de países industrializados de Europa y en los Estados Unidos, donde sus estrategias de atención propician una supervivencia a cinco años de $85 \%$ en los niños con leucemia linfoide aguda (8), cifra lejana para los niños colombianos, en quienes sólo llega al $41 \%(1)$.

Para el cumplimiento de este objetivo, la reflexión sobre las causas de la mortalidad por leucemias agudas pediátricas más allá de las causas clínicas, lleva a reconocer que el desconocimiento del protocolo por parte de los actores involucrados, la inobservancia de las actividades definidas en este y la falta de consolidación de los resultados de los comités de análisis de muerte por este hecho en el país, son aspectos que limitan el reconocimiento de las fallas en la atención y le resta argumentos a las entidades de control y tomadores de decisiones para exigir a las instituciones las acciones de mejora ante las deficiencias encontradas.

Por ende, si se busca reducir la mortalidad por este evento, es fundamental para el sistema de salud fortalecer el desarrollo de los comités de análisis, tener Instituciones Prestadoras de Servicios de Salud con infraestructura y recursos acordes con el nivel de complejidad y aplicar medidas de control a las Empresas Administradoras de Planes de Beneficios, con el fin de garantizar la calidad de la red prestadora de servicios para esta población $y$, por tanto, la equidad, accesibilidad, integralidad y continuidad en las atenciones requeridas durante su proceso de enfermedad, esto en concordancia con lo expuesto en la reciente Ley de Cáncer Infantil (29) y lo estipulado para la atención de la enfermedad como evento de alto costo (30); además, lo es involucrar a las familias y comunidades en programas de promoción y prevención, que informen sobre las señales de alarma generales para acudir a un centro médico e instaurar medidas para lograr la identificación oportuna de los casos (31).

Una limitación del presente estudio es que, aunque se incluyó la herramienta de investigación epidemiológica de campo, no fue posible su aplicación en todas las muertes debido al desconocimiento de roles y poca observancia del protocolo de vigilancia por parte de las instituciones responsables de esta actividad.

A pesar de esta limitación, los resultados presentados constituyen información importante para el desarrollo de los comités de análisis de la mortalidad por leucemias agudas pediátricas; en este sentido, se pone a disposición una experiencia 
más allá de las cifras estadísticas, buscando contribuir a mejorar el pronóstico y la supervivencia de los niños y las niñas con cáncer en el país.

\section{Agradecimientos}

Los autores agradecemos a los representantes del Instituto Nacional de Salud, de la Secretaría Distrital de Salud de Bogotá, de las Empresas Administradoras de Planes de Beneficios, de las Instituciones Prestadoras de Servicios de Salud y de las Empresas Sociales del Estado, que atendieron a la convocatoria de los comités de análisis de la mortalidad por leucemias agudas pediátricas. De igual forma, queremos agradecer a la Clínica de Oncología Pediátrica del Instituto Nacional de Cancerología por su asistencia y aportes en el desarrollo de las mismas.

\section{Conflicto de intereses}

Los autores declaramos que no tenemos conflictos de interés.

\section{Financiación}

El trabajo fue realizado con recursos de funcionamiento del Programa de Vigilancia Epidemiológica del Cáncer en el Instituto Nacional de Cancerología.

\section{Referencias}

1. Bravo LE, Collazos T, García LE, Gutiérrez A, Carrascal E. Cáncer infantil en Cali, Colombia, 1994-2003. Registro Poblacional de Cáncer en Cali. Cali: Camilo Torres Serna y Cia S.C.S; 2009.

2. Ferlay J, Shin HR, Bray F, Forman D, Mathera C, Parkin DM. Globocan 2008, cancer incidence and mortality worldwide: IARC CancerBase No. 10, International Agency for Research on Cancer. Fecha de consulta: 16 de mayo de 2011. Disponible en: http://globocan.iarc.fr

3. Belson M, Kingsley B, Holmes A. Risk factors for acute leukemia in children: A review. Environ Health Perspect. 2007;115:138-45. http://dx.doi.org/10.1289/ehp.9023

4. Centers for Disease Control and Prevention. Trends in childhood cancer mortality -United States, 1990-2004. MMWR Morb Mortal Wkly Rep. 2007;56:1257-61.

5. Bosetti C. Childhood cancer mortality in Europe, 1970-2007. Eur J Cancer. 2010;46:384-94. http://dx.doi.org/10.1016/j. ejca.2009.09.011

6. Couto AC, Ferreira JD, Koifman RJ, Monteiro GT, Pombode-Oliveira MS, Koifman S. Trends in childhood leukemia mortality over a 25-year period. J Pediatr. 2010;86:405-10. http://dx.doi.org/10.2223/JPED.2027

7. Instituto Nacional de Cancerología E.S.E. Cáncer en cifras: mortalidad por cáncer según primeras causas y sexo, tasas específicas por grupos de edad, Colombia, 2000-2008. Fecha de consulta: 16 de mayo de 2011. Disponible en: http:// www.cancer.gov.co/documentos/Mortalidad/Mortalidad\%20 nacional\%20por\%20tipo\%20de\%20cáncer\%20y\%20 grupo\%20de\%20edad\%202000-2008.pdf

8. Ribeiro RC, Pui $\mathbf{C H}$. Saving the children-improving childhood cancer treatment in developing countries. N Engl J Med. 2005;352:2158-60.

9. International Atomic Energy Agency. Inequity in cancer care: A global perspective. Vienna: IAEA; 2011.

10. Viana MB, Fernandes RA, de Oliveira BM, Murao M, de Andrade PC, Duarte AA. Nutritional and socio-economic status in the prognosis of childhood acute lymphoblastic leukemia. Haematologica. 2001;86:113-20.

11. Howard SC, Pedrosa M, Lins M, Pedrosa A, Pui CH, Ribeiro RC, et al. Establishment of a pediatric oncology program and outcomes of childhood acute lymphoblastic leukemia in a resource-poor area. JAMA. 2004;291:2471-5.

12. Howard SC, Metzger ML, Wilimas JA, Quintana Y, Pui CH, Robison LL, et al. Childhood cancer epidemiology in low-income countries. Cancer. 2008;112:461-72. http:// dx.doi.org/10.1002/cncr.23205

13. Congreso de la República de Colombia. Ley 1098 de 2006 (8 de noviembre) por la cual se expide el código de la Infancia y la Adolescencia. Diario Oficial No 46.446, 8 de noviembre de 2006. Bogotá D.C.: Congreso de Colombia; 2006.

14. Ministerio de la Protección Social. Circular 008 de 2008 por la cual se establece el sistema de vigilancia centinela de casos probables y confirmados de leucemias agudas pediátricas. 8 de febrero de 2008. Bogotá D.C.; Ministerio de la Protección Social; 2008.

15. Ministerio de la Protección Social. Decreto 3518 de 2006 por el cual se crea y reglamenta el Sistema de Vigilancia en Salud Pública y se dictan otras disposiciones. Diario Oficial No 46.417, 10 de octubre de 2006. Bogotá D.C.: Ministerio de la Protección Social; 2006.

16. Piñeros M, Pardo C, Otero J, Suárez A, Vizcaíno M, Álvarez $\mathrm{VH}$, et al. Protocolo de vigilancia en salud pública de las leucemias agudas pediátricas Rev Colomb Cancerol. 2007;11:219-27.

17. Alcaldía de Medellín. Modelos para el análisis de la mortalidad materna y perinatal. Medellín: Secretaría de Salud; 2005.

18. Instituto Nacional de Salud. Protocolo de vigilancia y control de la mortalidad por enfermedad diarreica aguda en menores de 5 años. 2009. Fecha consulta: 17 de mayo de 2011. Disponible en: http://www.minproteccionsocial.gov.co/ Documentos\%20y\%20Publicaciones/MORTALIDAD\%20 POR $\% 20$ EDA\%2 OMENORES\%20DE\% 205\% 20 A\%C3\%910S.pdf

19. Piñeros M, Pardo C, Otero J, Suárez A, Vizcaíno M, García $\mathrm{S}$, et al. Protocolo de vigilancia y control de las leucemias agudas pediátricas. 2010. Fecha de consulta: 3 de mayo de 2011. Disponible en: http://190.27.195.165:8080/index. php?idcategoria $=39005 \#$

20. Secretaría Distrital de Salud. Investigación epidemiológica de campo y estudio de brotes. 2011. Fecha de consulta: 3 de mayo 3 2011. Disponible en: http://www.saludcapital. gov.co/sitios/VigilanciaSaludPublica/Protocolos\%20de\%20 Vigilancia\%20en\%20Salud\%20Publica/Investig\%20de\%20 Campo\%20y\%20Estudio\%20Brotes.pdf 
21. Thaddeus S, Maine D. Too far to walk: Maternal mortality in context. Newsl Womens Glob Netw Reprod Rights. 1991;36:22-4.

22. Vélez G, Gómez J, Zuleta J. Análisis de las muertes maternas por hemorragia en el Departamento de Antioquia, Colombia. Años 2004 y 2005. Revista Colombiana de Obstetricia y Ginecología. 2006;57:147-55.

23. Instituto Nacional de Salud. Lineamientos de vigilancia y control en salud pública 2012. Fecha de consulta: 3 de mayo de 2011. Disponible en: http://www.ins.gov. co/?idcategoria $=93531 \#$

24. Curado MP, Pontes T, Guerra-Yi ME, Cancela MC. Leukemia mortality trends among children, adolescents, and young adults in Latin America. Rev Panam Salud Pública. 2011;29:96-102. http://dx.doi.org/10.1590/S102049892011000200004

25. Wilkins KL, Woodgate RL. An interruption in family life: Siblings' lived experience as they transition through the pediatric bone marrow transplant trajectory. Oncol Nurs Forum. 2007;34:E28-35.

26. Woodgate RL, Degner LF. Cancer symptom transition periods of children and families. J Adv Nurs. 2004;46:35868. http://dx.doi.org/10.1111/j.1365-2648.2004.03002.x
27. Belizán JM, Cafferata ML, Belizán M, Althabe F. Health inequality in Latin America. Lancet. 2007;370:1559-600. http://dx.doi.org/10.1016/S0140-6736(07)61673-0

28. La VC, Levi F, Lucchini F, Lagiou P, Trichopoulos D, Negri E. Trends in childhood cancer mortality as indicators of the quality of medical care in the developed world. Cancer. 1998;83:2223-7.

29. Congreso de la República de Colombia. Ley 1388 de 2010 (26 de mayo) por el derecho a la vida de los niños con cáncer. Diario Oficial No 47.721, 26 de mayo de 2010. Bogotá D.C.: Congreso de la República; 2010.

30. Ministerio de la Protección Social. Resolución 003974 de 2009 (21 de octubre) por la cual se adoptan unas determinaciones en relación con la cuenta de alto costo. Diario Oficial No 47.516, 28 de octubre de 2009. Bogotá D.C.: Ministerio de la Protección Social; 2009.

31. Organización Panamericana de la Salud. Estrategias de comunicación para la salud integral en la infancia: guía metodológica para su desarrollo. Washington, D.C.: OPS; 2005. 